\title{
BEARING FAULT DETECTION USING ACOUSTIC EMISSION SIGNALS ANALYZED BY EMPIRICAL MODE DECOMPOSITION
}

\author{
Niranjan Hiremath $^{1}$, D.Mallikarjuna Reddy ${ }^{2}$ \\ ${ }^{1}$ Assistant Prof., Dept. of Mechanical Engg., Reva Institute of Technology and Mgmt. Bangalore, Karnataka, India \\ ${ }^{2}$ Associate Prof., Dept. of Mechanical Engg., Reva Institute of Technology and Mgmt. Bangalore, Karnataka, India
}

\begin{abstract}
In condition monitoring of ball bearings, traditional techniques involving vibration, acceleration may not be able to detect a growing fault due to the low impact energy generated by the relative motion of the components. This study presents an experimental evaluation for incipient fault detection of lightly loaded ball bearings by using acoustic emission method. A table top bearing test rig is fabricated to simulate bearing faults such as inner race \& outer race defect etc., with rotating shaft carrying low loads. The effectiveness of the acoustic analysis technique is demonstrated through signal processing techniques; use of Empirical Mode Decomposition (EMD) method to derive the characteristics features related to defective bearings from time domain and enveloped spectra in the frequency domain. The results reveal that the acoustic technique is more effective in detecting bearings' failure than that of the vibration measurement.
\end{abstract}

Keywords: Condition Monitoring, Acoustic Emission, Vibration Analysis, Empirical Mode Decomposition.

\section{INTRODUCTION}

Condition Monitoring (CM) is the process used to determine the operational state and health of a machine for the purpose of detecting potential failures before they turn into functional failures. The CM process consists of periodical or continuous data collection, data analysis, interpretation and diagnosis. Condition Monitoring is an integral part of Predictive Maintenance (PM) which is a widely used maintenance philosophy (also known as Condition-Based Maintenance CBM). This philosophy is based on scheduling maintenance activities only when a possible functional failure is detected. CBM enhances operational effectiveness and safety through research and development of technologies to accurately and reliably predict the remaining useful life of in-service equipment. CM optimizes equipment readiness while reducing maintenance and staffing requirements. Typical CM techniques include vibration analysis, oil analysis, wear particle analysis, ultrasonic analysis, thermographic analysis and motor current signature analysis $[1,2]$.

One of the more popular tools in the condition monitoring of rotating machinery is vibration analysis. By measurement and analysis of the vibration in rotating machinery, it is possible to detect typical faults such as unbalance, bent shaft, cracked shaft, misalignment, looseness, rubbing, gear faults, motor faults and impellor/blade defects. However, these vibration based methods are effective when the defect in the bearings has already become severe. But the vibration signal is not sensitive to the incipient defect. Furthermore, the vibration signal caused by bearing defects will always be contaminated and distorted by other faults and mechanical noise. Hence, it is difficult for the vibration signal based methods to detect bearing defects at an early stage, and a more sensitive detection and diagnosis technique to provide an early and unambiguous indication of bearing defects would be extremely valuable. There is limited information on faults which occur exclusively in low speed machinery, other than those initiated by rolling element bearing defects [3].

Numerous research works have been published on the detection and diagnosis of rolling element bearing defects. Tandon and Nakra [4] presented a detailed study on vibration measurements in the time and frequency domains and the shock pulse method. Tandon and Choudhury [5] subsequently updated the reviews incorporating more recent works and advanced techniques currently being adopted in bearing defect detection. Most of the research on bearing diagnosis can be categorized in the time domain and frequency domains. The RMS, crest factor, probability density moments (skewness, kurtosis) are the most popular statistical time domain parameter for bearing defect detection [6-10]. In the frequency domain, the enveloping method, also known as demodulation or HFRT (High Frequency Resonance Technique), have been proven to be a very efficient and popular technique for detection of the characteristic frequencies of bearings [11-16]. Wavelet analysis has also been successfully applied to bearing defect detection [17-21]. In recent years, artificial neural networks and fuzzy logic have also emerged as popular tools for automated fault diagnosis [18, 22-25].

In this study, the effectiveness of the acoustic emission technique for condition monitoring of low speed bearings is 
emphasized using empirical mode decomposition method. An artificially induced fault in the form of a minute scratch on the bearing inner-race and outer-race was utilized for the study. The acoustic signal from a good bearing and defective bearings are compared with vibration signals in frequencydomain envelop spectrum at shaft speed of $1200 \mathrm{rpm}$.

\section{EMPIRICAL MODE DECOMPOSITION METHOD (EMD)}

The signal processing method based on Hilbert- Huang Transform is considered a great breakthrough of linear and stationary and non- stationary signal. For instantaneous frequency method owning physics sense is not applicable to arbitrary signal except mono component signal, where as the signals such as nature and engineering field obtained commonly do not satisfy the mono- component signal requirement. So only EMD is needed to decompose the signal into a series of mono-component contributions designated as IMF (Intrinsic Mode Function), namely:

$$
f(t)=\sum_{j=1}^{n} c_{j}(t)+r n
$$

Where $r_{n}$ is the residue after the ' $\mathrm{n}$ ' IMFs have been extracted?

In general, the first IMFs contain the finest scale or the shortest period component of the signal and can be either the mean trend or a constant. Each characteristic oscillatory mode extracted, named IMF and satisfies the following properties: the IMFs are characterized by having the number of extrema and the number of zero crossings equal or differing at most by one, and the mean value between the upper and low envelope equal to zero ay any point. In other words, an IMF is symmetric. It has a unique local frequency and different IMFs do not exhibit the same frequency at the same time. The IMF satisfied above condition namely is a mono-component signal. The character of IMF also is convergence standard of EMD decomposition end.

The EMD decomposition is developed from the simple assumption that any complicated signal $f(t)$ consists of different simple intrinsic modes of oscillation. Having obtained the IMF components, one will have no difficulty in applying the Hilbert transform to each of the IMF components and computing the instantaneous frequency to get Hilbert spectrum. Given

$$
x_{j}(t)=c_{j}(t)
$$

For $x_{j}(t)$, the Hilbert transform reads

$$
y_{j}(t)=\frac{1}{\pi} P \cdot V \cdot \int_{-\infty}^{\infty} \frac{x_{j}(\tau)}{t-\pi} d \pi
$$

With P.V. meaning the Cauchy principal value of this integral $x_{j}(t)$ and $y_{j}(t)$ form a complex conjugate pair, which defines an analytic signal $z_{j}(t)$ as

$$
z_{j}(t)=x_{j}(t)+i y_{j}(t)=a_{j}(t) e^{i \theta_{j}(t)}
$$

With amplitude $a_{j}(t)$ and phase $\theta_{j}(t)$ defined by the expression

$$
a_{j}(t)=\left[x_{j}^{2}(t)+y_{j}^{2}(t)\right]^{1 / 2}
$$

And

$$
\theta_{j}(t)=\arctan \left(\frac{y_{j}(t)}{x_{j}(t)}\right)
$$

$a_{j}(t)$ is named as the instantaneous envelopment.

Instantaneous frequency of $x_{j}(t)$ is defined as

$$
\omega_{j}(t)=\frac{d \theta_{j}(t)}{d t}
$$

Thus, the original data can be expressed in the following form:

$$
f(t)=R P \sum_{j=1}^{n} a_{j}(t) \exp \left(i \int \omega_{j}(t) d t\right)
$$

Whereas the residue $r_{n}$ is left out, because it is a monotonic function (data trend), or a constant

This frequency time distributions of the amplitude is designed as the Hilbert-Huang spectrum $H(\omega, t)$. So energy spectrum reflects frequency component in a certain time locality contribution to signal.

\section{ACOUSTIC EMISSION (AE)}

Acoustic emission is a transient elastic wave generated when strain energy is suddenly released due to the relative motion 
among the particles or material at a small scale. The small scale of the $\mathrm{AE}$ generation mechanisms mean that the frequencies generated are usually very high, although the source mechanisms are usually smaller than for vibration. Examples include the material fracture in crack propagation or the interaction of asperities in sliding surfaces.

$\mathrm{AE}$ has been considered for many years as a prime candidate for the nondestructive examination, testing, or monitoring (NDT) of material faults or structural failure and has found broad applications in these fields. When a rolling element bearing with defects operates, the defect (surface defect, cracking, spalling, etc.) will generate elastic impulses and strain on the contact surface between the roller and the race of the bearing. This will lead to a release of transient stress wave energy, that is, AE. This AE contains direct and abundant information about the bearing defect and thus could be used to effectively detect and diagnose such defects. In contrast to the vibration signal, $\mathrm{AE}$ generally covers a frequency range over $20 \mathrm{kHz}$ and does not exhibit a significant spectral overlap with mechanical vibration signals from rotating machinery. This means that $\mathrm{AE}$ is not influenced or disturbed by other mechanical noise, and faults in rotating machinery, such as imbalance and misalignment, which cannot be eliminated easily and completely. Thus AE based methods are superior in some areas to vibration based methods for defect detection, especially for incipient defect detection in rolling element bearings.

\section{BEARING FUNDAMENTAL FREQUENCIES}

The frequencies that rolling element bearings generate when rollers pass over a surface anomaly on either the roller or the raceway are called fundamental fault frequencies. These frequencies are a function of the bearing geometry (that is pitch diameter and roller diameter) and the relative speed between the two raceways. When bearing geometry is known, the fundamental fault frequencies can be calculated using Equations 9 to 12 in $\mathrm{Hz}$.

$$
\begin{aligned}
& \mathrm{BPFI}=(\mathrm{N} / 2) \times \mathrm{F}(1+(\mathrm{B} / \mathrm{P} \times \cos \Theta)) \\
& \mathrm{BPFO}=(\mathrm{N} / 2) \times \mathrm{F}(1-(\mathrm{B} / \mathrm{P} \times \cos \Theta)) \\
& \mathrm{FTF}=(\mathrm{F} / 2)(1-(\mathrm{B} / \mathrm{P} \times \cos \Theta)) \\
& \mathrm{BPF}=(\mathrm{P} / 2 \mathrm{~B}) \times \mathrm{F}\left(1-(\mathrm{B} / \mathrm{P} \times \cos \Theta)^{2}\right)
\end{aligned}
$$

Where,

$$
\begin{aligned}
& \text { BPFI }=\text { Ball Pass Frequency Inner race }(\text { in } \mathrm{Hz}) \\
& \text { BPFO = Ball Pass Frequency Outer race (in Hz) } \\
& \text { FTF }=\text { Fundamental Train Frequency }(\text { in } \mathrm{Hz}) \\
& \text { BPF }=\text { Ball Pass Frequency (in } \mathrm{Hz} \text { ) } \\
& \mathrm{N}=\text { number of balls } \\
& \mathrm{F}=\text { Shaft frequency }(\text { in } \mathrm{Hz})
\end{aligned}
$$

$$
\begin{aligned}
& \mathrm{B}=\text { Ball Diameter }(\text { in } \mathrm{mm}) \\
& \mathrm{P}=\text { Pitch Diameter }(\text { in } \mathrm{mm}) \\
& \Theta=\text { Contact angle }
\end{aligned}
$$

\section{EXPERIMENTAL SETUP}

The test rig used in this study is as shown in Fig-1. The testrig consists of a shaft with a dead weight in the form of a disc. The shaft is turned to the designed diameter on the precision lathe. The Shaft is supported by two deep groove ball bearings (SKF6304-2Z) and connected to a dimmer stat speed controlled induction motor through a flexible coupling. Two bearing seating on the shaft are ground and hardened.

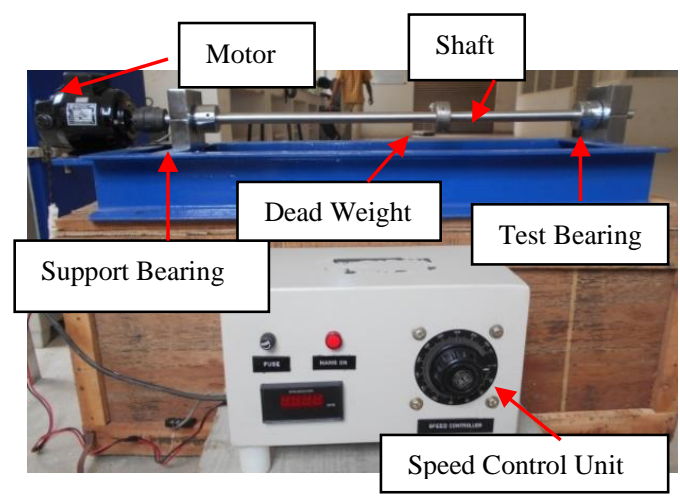

Fig-1: Table top bearing test rig

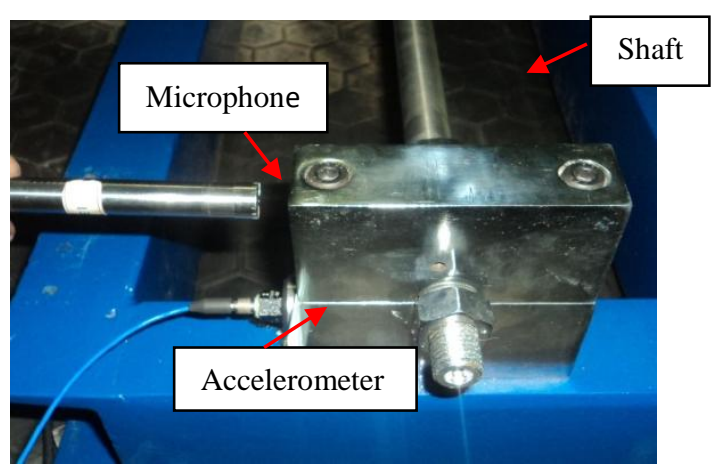

Fig-2: Location of microphone and accelerometer near the test bearing

At one end there is self-aligning ball bearing and at the other end there is one more ball bearing which is to be tested. The disc is mounted on the shaft at a distance $2 / 3 \mathrm{rd}$ of total length of shaft from support bearing and is fit on the shaft by two grub screws, screwed radically on the disc. The ball bearing is tested at constant load (400gms) and at constant speed of 710rpm. The localized defect was obtained by a minute scratch on the outer race and inner race of the test bearing. Previous studies have also involved seeded defects induced by using acid etching, spark erosion, scratching or mechanical indentation. Experiments are carried out with bearing test rig 
by positioning the sensors near the test bearing as shown in Fig-2.

The Specifications of the test bearing used in the experiment are tabulated in Table 1 and the bearing used is shown in Fig3.

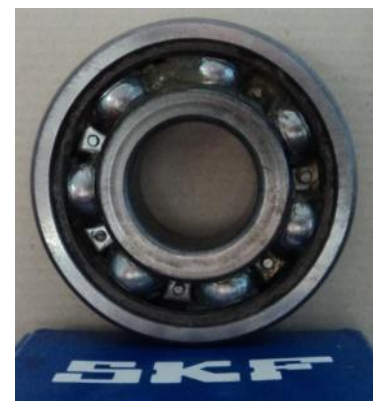

Fig-3: Test Bearing

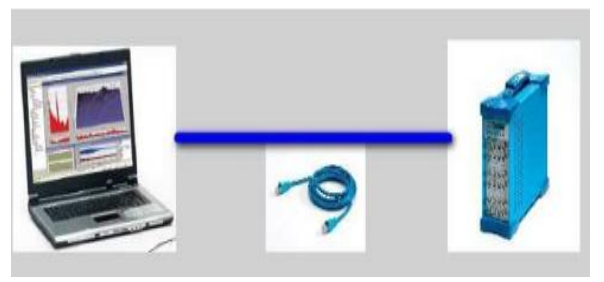

Fig-4: Data acquisition system used for capturing signals

Navigate v.6 is the instrument used for carrying out vibration and acoustic testing which is as shown in Fig-4. It consists of piezo electric transducer (AC 750, 4525) with resonant frequency of $750 \mathrm{KHz}$ which senses the vibration and acoustic wave and converts it into electrical voltage which is sent to the main frame for processing through a pre-amplifier.

Table-1: Specifications of the test bearing.

\begin{tabular}{|l|l|}
\hline Feature & Detail \\
\hline Bearing Type & SKF6304-2Z \\
\hline Number of Balls & 7 \\
\hline Outside diameter & $52 \mathrm{~mm}$ \\
\hline Inside diameter & $20 \mathrm{~mm}$ \\
\hline Pitch diameter & $36 \mathrm{~mm}$ \\
\hline Ball Diameter & $09 \mathrm{~mm}$ \\
\hline
\end{tabular}

\section{RESULTS AND DISCUSSIONS}

\subsection{Vibration Analysis in Frequency Domain}

Vibration signals are captured from the test bearing with good, outer race defect and inner race defect conditions. These signals are then superimposed as shown in Fig-5.

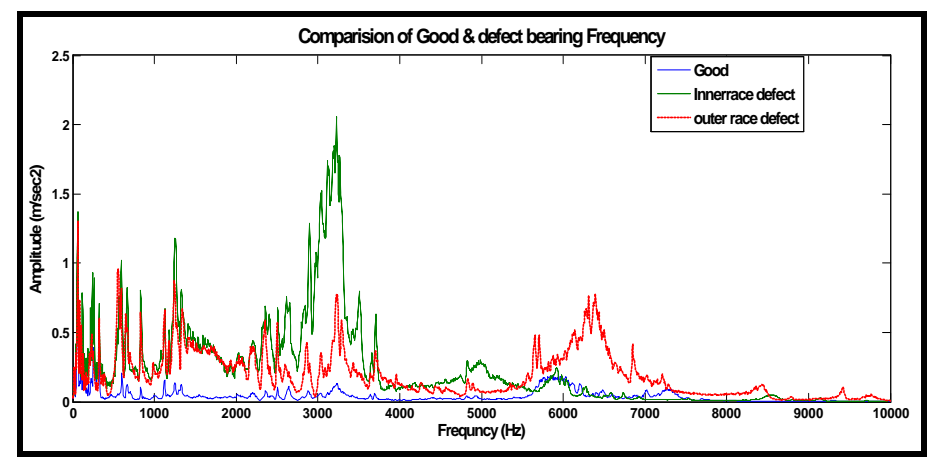

Fig-5: Variation of frequency pattern for bearing with good, outer race defect and inner race defect conditions.

Frequency versus amplitude plot showed in Fig-5 shows very small amplitude values for good bearing comparing with both defect cases. It clearly shows that signal of inner race defect is completely dominating compared to other cases and there is increase in amplitude of around 1.5 times compared with good bearing throughout the frequency domain data.

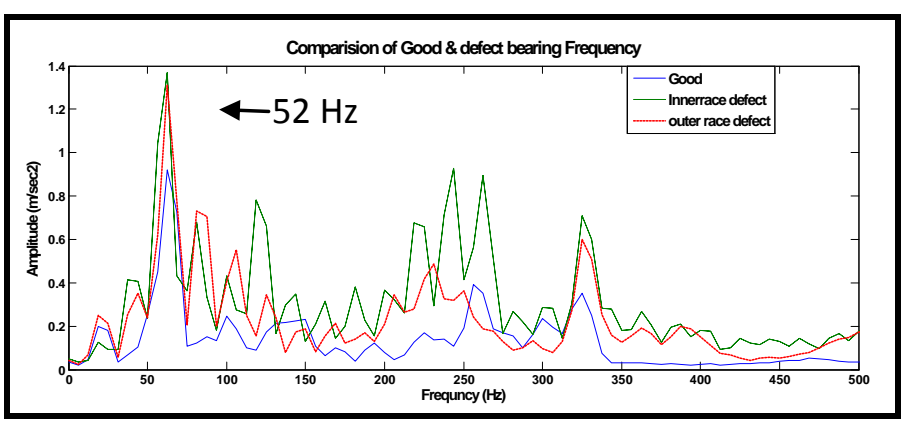

Fig-6: Variation of frequency pattern (zoomed between 0 to $500 \mathrm{~Hz}$ ) for bearing with good, inner race defect and outer race defect conditions.

Frequency versus amplitude plot shown in Fig-6 shows the zoomed view between 0 to $500 \mathrm{~Hz}$. It is well known that inner race defects are more difficult to detect than outer race defects due to the relative motion of the ring and the ball elements. Hence, the bearing characteristic frequency for inner race fault (BPFI) is calculated using Eq-9 and it is found to be equal to approximately $52 \mathrm{~Hz}$. Therefore, there is sudden peak in the amplitude at this frequency as it is evident in Fig-6 and also harmonics of the inner race defect are clearly visible and dominated throughout the frequency domain.

\subsection{Acoustic Analysis}

Using Microphone, raw acoustic signals are obtained for good, outer race defect and inner race defect conditions of bearing. These signals are then processed using EMD Algorithm to obtain IMF's as shown in Fig-7, Fig-8 and Fig-9. The MATLAB software was used for the purpose. 


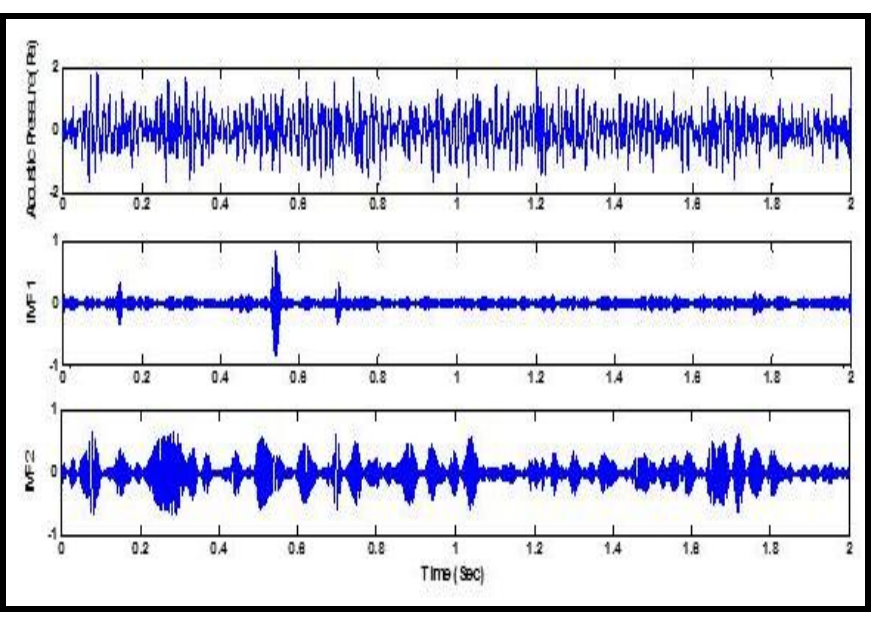

Fig-7: (From top) (i) Raw acoustic time history response data for good bearing (ii) IMF 1 for good bearing (iii) IMF 2 for good bearing.

Acoustic signals of good bearings are shown in Fig-7. In Fig7, the IMF's clearly show that there is no sudden continuous spike along the length of the signal.

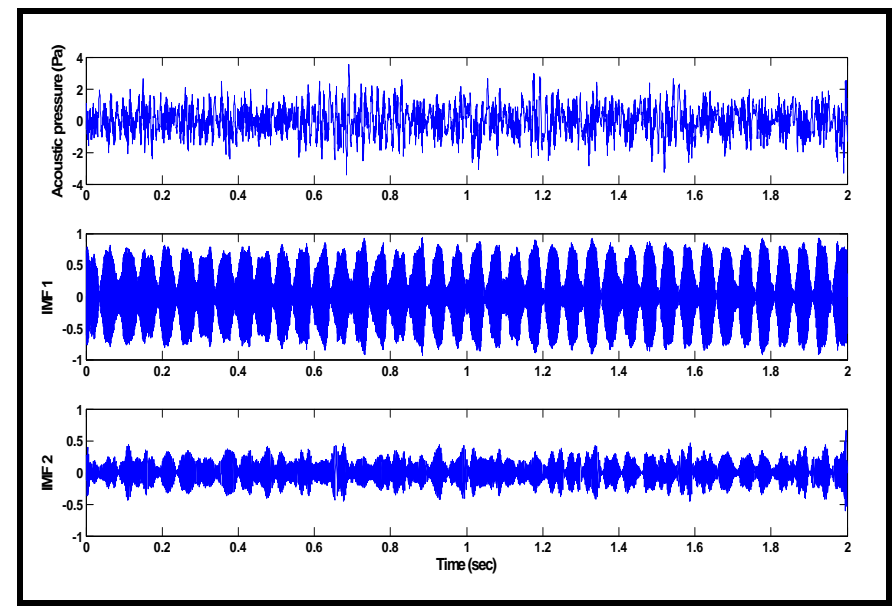

Fig-8: (i) Raw acoustic time history response data for bearing with outer race defect (ii) IMF 1 for outer race defect bearing

(iii) IMF 2 for outer race defect bearing.

Acoustic signals of outer race defect bearing are shown in Fig8. By comparing raw signals of good bearing (Fig-7) and outer race defect bearing (Fig-8) there is no information regarding the defect in the bearings. To find the location of defect in the bearing, raw defect data is processed into EMD algorithm to obtain IMFs' produced. In Fig-8, IMF 1 clearly shows continuous spikes along the length of the signal due to crack or defect in the outer race. IMF 2 is not sensitive for the defect in the bearing as clearly shows low amplitudes of spikes.

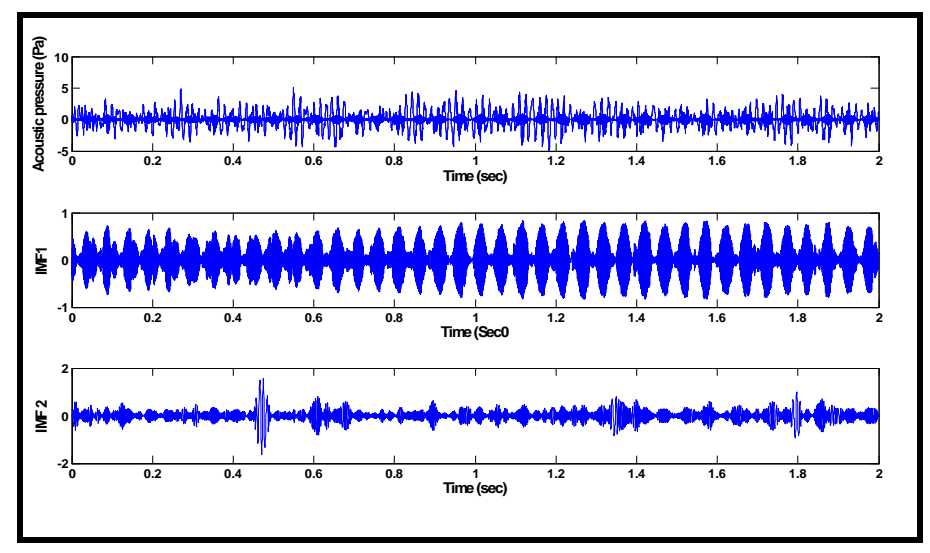

Fig-9: (i) Raw acoustic time history response data for bearing with inner race defect (ii) IMF 1 for inner race defect bearing (iii) IMF 2 for inner race defect bearing.

Acoustic Signals of Inner Race Defect Bearing are shown in Fig-9. In Fig-9, IMF 1 clearly shows continuous spikes along the length of the signal due to crack or defect in the inner race.

\section{CONCLUSIONS}

A number of experiments have been carried out to investigate the effectiveness of the acoustic technique for condition monitoring of low speed bearings. The defective bearings with seeded defects on the inner race and outer race were used in conjunction with a healthy bearing. The vibration signals obtained from an accelerometer were also measured and analyzed for comparative purposes. This study revealed that inner race frequency amplitudes are dominating in the overall frequency domain when compared with good and outer race defect conditions. Empirical Mode Decomposition algorithm is better technique to detect the defects in the bearings and also used to estimate the severity of damage. IMFs will give better estimation of defect sensitive for frequency. It is evident from the results that Acoustic signal trends can be correlated with bearing analysis which establishes a better understanding of individual machine bearing characteristics. Present work highlighted the procedure for identification of defect in the bearing. But, further study can be carried out for finding exact location of defects in the bearing.

\section{ACKNOWLEDGEMENTS}

We acknowledge Envicon Vibrotech Instruments firm for providing facilities to carry out series of experiments.

\section{REFERENCES}

[1]. R. James, "Machine Condition Monitoring...A Catalist for Change in Industrial Equipment Maintenance," P/PM Technology, vol. October, pp. 20-40, 2001.

[2]. R. James, "Machine Condition Monitoring...A Catalist for Change in Industrial Equipment Maintenance," P/PM Technology, vol. December, pp. 18-37, 2001. 
[3]. J. E. Berry, "Required Vibration Analysis Techniques and Instrumentation on Low Speed Machines": Technical Associates of Charlotte, PC, 1997.

[4]. N. Tandon and B. C. Nakra, "Vibration and acoustic monitoring techniques for the detection of defects in rolling element bearings - a review," Shock and Vibration Digest, vol. 24, pp. 3-11, 1992.

[5]. N. Tandon and A. Choudhury, "A review of vibration and acoustic measurement methods for the detection of defects in rolling element bearings," Tribology International, vol. 32, pp. 469-480, 1999.

[6]. S. Braun and B. Datner, "Analysis of Roller/Ball Bearing Vibrations," Transactions of the ASME, vol. 101, pp. 118-125, 1979.

[7]. R. J. Alfredson and J. Mathew, "Time Domain Methods for Monitoring the Condition of Rolling Element Bearings," presented at Mechanical Engineering Transactions, 1985.

[8]. D. Dyer and R. M. Stewart, "Detection of Rolling Element Bearing Damage by Statistical Vibration Analysis,"

Journal of Mechanical Design, Transaction of the ASME, vol. 100, pp. 229-235, 1978.

[9]. J. Mathew and R. J. Alfredson, "The Condition Monitoring of Rolling Element Bearings Using Vibration Analysis," Journal of Vibration, Acoustics, Stress, and Reliability in Design, Transaction of ASME, vol. 106, 1984.

[10]. N. Tandon and B. C. Nakra, "Detection of Defects in Rolling Element Bearings by Vibration Monitoring," IE(I)Journal MC, vol. 73, pp. 271-282, 1993.

[11]. T. I. Liu and J. M. Mengel, "Intelligent Monitoring of Ball Bearing Conditions," Mechanical Systems and SignalProcessing, vol. 6, pp. 419-431, 1992.

[12]. R. J. Alfredson and J. Mathew, "Frequency Domain Methods for Monitoring the Condition of Rolling Element

Bearings," presented at Mechanical Engineering Transactions, 1985.

[13]. J. I. Taylor, "Identification of Bearing Defects by Spectral Analysis," Journal of Mechanical Design, Transaction of the ASME, vol. 102, pp. 199-204, 1980.

[14]. P. D. McFadden and J. D. Smith, "Vibration monitoring of rolling element bearings by the high frequency resnance technique - a review," Tribology International, vol. 17, pp. 310, 1984.

[15]. Y. A. Azovtsev, A. V. Barkov, and L. A. Yudin, "Automatic Diagnostics and Condition Prediction of Rolling Element Bearings Using Enveloping Methods," presented at Vibration Institute Proceedings, 18th Annual Meeting, Hershey, Pennsylvania, June 21-23., 1994.

[16]. A. V. Barkov, N. A. Barkova, J. S. Mitchell, and S. J. Capistrano, "Assessing the Condition and Lifetime of Rolling Element Bearings from a Single Measurement," presented at Vibration Institute Proceedings, 19th Annual Meeting, Indianapolis, Indiana, June 20-22, 1995.

[17]. J. Lin, M. J. Zuo, and K. R. Fyfe, "Mechanical Fault detection Based on the Wavelet De-Noising Technique," Journal of Vibration and Acoustics, Transactions of the ASME, vol. 126, pp. 9-16, 2004.
[18]. B. A. Paya, I. I. Esat, and M. N. M. Badi, "Artificial Neural Network Based Fault Diagnostics Of Rotating Machinery Using Wavelet Transforms As A Preprocessor," Mechanical Systems and Signal Processing, vol. 11, pp. 751$765,1997$.

[19]. J. Lin and L. Qu, "Feature Extraction Based On Morlet Wavelet And Its Application For Mechanical Fault Diagnosis," Journal of Sound and Vibration, vol. 234, pp. 135$148,2000$.

[20]. P. W. Tse, Y. H. Peng, and R. Yam, "Wavelet Analysis and Envelope Detection For Rolling Element Bearing Fault Diagnosis - Their Effectiveness and Flexibilities," Journal of Vibration and Acoustics, Transactions of the ASME, vol. 123, pp. 303-310, 2001.

[21]. B. Liu, S. F. Ling, and R. Gribonval, "Bearing failure detection using matching pursuit," NDT \& E International, vol. 35, pp. 255-262, 2002.

[22]. M. Subrahmanyam and C. Sujatha, "Using neural networks for the diagnosis of localized defects in ball bearings," Tribology International, vol. 30, pp. 739-752, 1997.

[23]. B. Li, G. Goddu, and M.-Y. Chow, "Detection of common motor bearing faults using frequency-domain vibration signals and a neural network based approach," presented at American Control Conference, 1998. Proceedings of the $1998,1998$.

[24]. C. K. Mechefske, "Objective Machinery Fault Diagnosis Using Fuzzy Logic," Mechanical Systems and Signal Processing, vol. 12, pp. 855-862, 1998.

[25]. B. Li, M.-Y. Chow, Y. Tipsuwan, and J. C. Hung, "Neural-network-based motor rolling bearing fault diagnosis," Industrial Electronics, IEEE Transactions on, vol. 47, pp. 1060-1069, 2000. 